\title{
Fatores Associados ao Domicílio e à Família na Determinação da Hanseníase, Rio de Janeiro, Brasil
}

\author{
Factors Associated with Household and Family in Leprosy Transmission \\ in Rio de Janeiro, Brazil
}

\author{
Vera Lúcia G. de Andrade ${ }^{1}$ \\ Paulo C. Sabroza ${ }^{2}$
}

Adauto José G. de Araújo²

\begin{abstract}
ANDRADE, V.L. G.; SABROZA, P. C. E ARAÚfO, A. F. G. Factors Associated with Household and Family in Leprosy Transmission in Rio de Faneiro, Brazil. Cad. Saúde Públ., Rio de Faneiro, 10 (supplement 2): 281-292, 1994.

A cross-sectional study was carried out to characterize the contribution of several household characteristics to the transmission of leprosy. Households with diagnosed cases of illness were compared to two healthy groups. All randomly selected households were located in the municipality of Sao Goncalo, in the State of Rio de Faneiro. Using an aerial map with the description of the census tract, 2,412 cases were marked. Three groups were established, while the household was the analytical unit: group I - households with cases; group II - neighboring households; and group III - households located in tracts with no reported case of illness, that is, outside disease foci. Group I was compared with that of neighboring households using a multiple logistic regression model by conditional methods. Unconditional methods were used to compare groups I and III. Group I as compared to Group II showed an association with age and educational level for households and heads of families. Comparison of characteristics of the heads of families and households with cases of leprosy with those located outside the focus showed that the differential factors were age, type of dwelling, and availability of running water. This is probably due to more recent settlement in a peripheral region where water resources are not available yet. Households are the basic ecological unit, and age and educational level are determinant factors for leprosy morbidity in this area.
\end{abstract}

Key words: Leprosy; Urban Areas; Human Dwellings; Epidemiology; Cross-Sectional Studies

\section{INTRODUÇÃO}

Segundo os resultados de indicadores epidemiológicos, o Brasil ainda apresenta altas taxas de detecção de hanseníase, assim como elevados coeficientes de prevalência, ao contrário da tendência global, cujos indicadores de transmissão apontam comportamento decrescente da enemia. Em algumas áreas do país, a hanseníase

1 Instituto Estadual de Dermatologia Sanitária da Secretaria do Estado do Rio de Faneiro. Rua Godofredo Viana, 64, Rio de Faneiro, RF, 22730-020, Brasil.

2 Escola Nacional de Saúde Pública, Fundação Oswaldo Cruz. Rua Leopoldo Bulhões, 1480, Rio de Faneiro, RF, 21041-210, Brasil. mostra magnitudes equivalentes às mais altas do mundo, mesmo quando comparadas com alguns países da África e Ásia.

Diversos estudos dos prováveis determinantes da hanseníase foram realizados a partir de 1936, acompanhando a produção científica no campo da epidemiologia desde a análise da distribuição dos atributos, definição dos indicadores e do conceito de risco até a utilização de modelos e técnicas estatísticas mais recentes, por meio do aperfeiçoamento dos desenhos de pesquisa e da utilização intensiva da informática na análise dos dados (Doull et al., 1936; Burgos, 1939; Guinto \& Rodrigues, 1941; Doull et al., 1942; Bancroft et al., 1944; Del Favero, 1948; Martins de Barros et al., 1960; Quagliato 
et al., 1963; Rao et al., 1969, 1972; Marshall et al., 1981; Chatuverdi, 1988; Ponnighaus et al., 1987; Maine et al., 1988; George et al., 1990).

No Brasil, não foram até hoje desenvolvidos estudos do tipo longitudinal, visando à identificação dos fatores associados. Entretanto, na busca de determinantes dessa doença, quatro grandes estudos transversais, a partir da década de 1940, contribuíram de forma importante para o conhecimento epidemiológico da endemia (Del Favero, 1948; Martins de Barros et al., 1960; Quagliato et al., 1963).

Data de 190 a.C. a sugestão de algumas características das relações das pessoas com seu habitat como possíveis causas da hanseníase, como aglomeração, promiscuidade e falta de higiene (Browne, 1985). Doull et al. (1936), pela primeira vez, suscitam questões concernentes ao modo de vida da população e sua condição geral de nutrição como fatores associados à alta incidência da hanseníase. A partir da década de 1940, Bechelli (1949) demonstra que as áreas de maior prevalência de hanseníase apresentam a maior parte da população vivendo em locais com serviços de saúde inadequados e condições sanitárias precárias. Isso foi confirmado por Trautman (1984), que discute a coincidência do aumento da qualidade de vida, traduzida pelas condições sócio-econômicas, com o declínio dos indicadores de magnitude da endemia em diversos países, chegando, em alguns casos, até mesmo à interrupção da transmissão.

Desse modo, a freqüência da hanseníase, assim como da tuberculose, malária, doença de Chagas e leishmaniose, passou a ser um parâmetro para diferenciação das condições sociais entre países. Entretanto, os comportamentos atuais de diversas endemias no Brasil não podem ser mais exclusivamente explicados mecanicamente pelas etapas do processo de desenvolvimento econômico (Sabroza, 1991).

Este trabalho tem por objetivo analisar algumas características dos domicílios e dos chefes de família como possíveis fatores de determinação da hanseníase. Compararam-se unidades domiciliares onde existiam casos de hanseníase com unidades domiciliares de não hansenianos, domicílios vizinhos dos doentes e os domicílios fora dos focos ou áreas sem casos de hanseníase. Todos os domicílios localizam-se em uma mesma área endêmica urbana, na periferia da região metropolitana do Rio de Janeiro.

\section{MATERIAL E MÉTODOS}

Em um mapa aerofotogramétrico do município de São Gonçalo, região metropolitana do Estado Rio do Janeiro, na escala de 1:120.000, fornecido pela Fundação Instituto Brasileiro de Geografia e Estatística (IBGE) e contendo a descrição dos 584 setores censitários, marcaram-se todos os 2412 casos diagnosticados residentes no município desde 1927 até dezembro de 1987. Essa localização foi feita segundo os setores censitários e as ruas correspondentes ao endereço fornecido pelas Unidades Sanitárias. Cinco por cento dos setores não fizeram parte do inquérito por serem setores de áreas militares e de segurança nacional (Figura 1).

A população residente no município de São Gonçalo era de 615.351 habitantes, exclusivamente urbana e apresentando densidade demográfica de 2.700 habitantes $/ \mathrm{km}^{2}$. As características geopolíticas, os parâmetros relacionados à hanseníase (Andrade, 1990), bem como a metodologia mais detalhada do inquérito epidemiológico realizado nesse município, já foram descritos anteriormente (Andrade et al., 1990).

A seleção dos domićlios com caso de hanseníase foi resultado do sorteio sistemático de um em cada cinco casos da relação nominal de todos os 1676 casos prevalentes em dezembro de 1987, listados por ordem da data de detecção. Para compor a amostra de domicílios sem casos de hanseníase adotaram-se quatro casas vizinhas a cada domicílio de doentes e cinco domicílios em setores onde não havia caso de hanseníase conhecido ou registrado até o momento do mapeamento. O número de unidades domiciliares sorteadas nesses setores foi proporcional ao número total de domicílios. Para selecionar os incluídos no estudo, sorteouse uma rua, numeraram-se os domicílios e, finalmente, sorteou-se o domicílio nuclear a ser pesquisado.

A realização de visita de domicílio por domicílio e o mapeamento dos casos de hanseníase, que permitiu a visualização dos setores e ruas como um mosaico de focos e setores in- 
FIGURA 1. Distribuição dos Casos de Hanseníase segundo os Setores Censitários, São Gonçalo, Rio de Janeiro, Brasil, 1927-1987

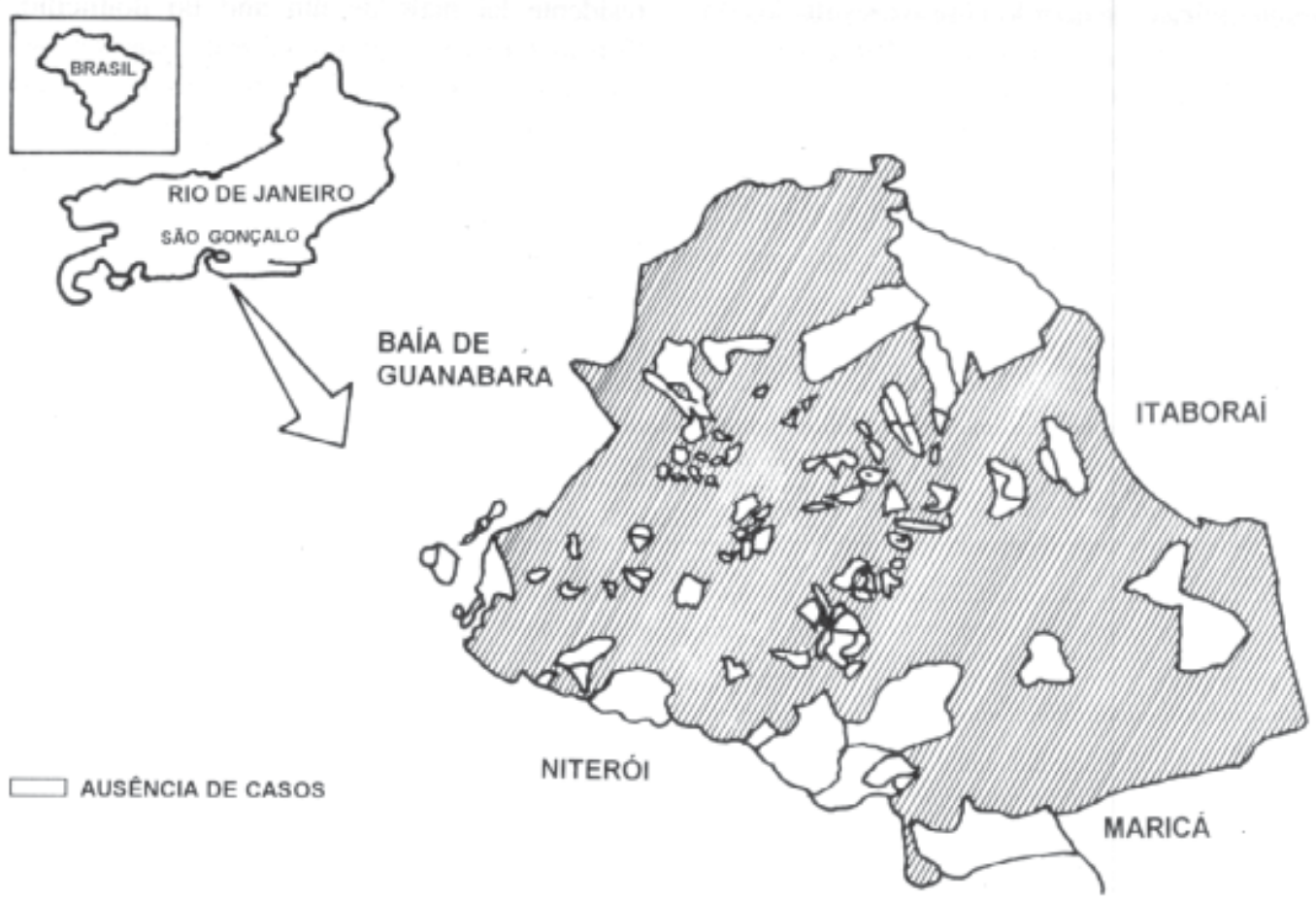

denes, somente foram possíveis, nessa escala, utilizando etapas metodológicas já proposta por outros autores (Doull et al., 1936; Burgos, 1939; Guinto \& Rodrigues, 1941; Del Favero, 1948; Marshall et al., 1981).

Para a realização do trabalho de campo, foi constituída uma equipe de investigadores composta por 20 técnicos do sistema de saúde e 16 comunitários. No período de 15 dias que antecederam o trabalho de campo, realizou-se um treinamento visando a padronização do preenchimento do questionário e do exame clínico. Iniciou-se o inquérito enviando-se uma carta aos domicílios selecionados, esclarecendo os motivos da visita. Os investigadores receberam o questionário apenas com o endereço que as Unidades Sanitárias forneceram, sem saber se os domicílios eram ou não de caso de hanseníase. A lista dos pacientes com esse dado só era do conhecimento dos supervisores.
O inquérito foi realizado durante 85 dias no período de agosto a novembro de 1988. Cada domicílio amostrado foi visitado no mínimo duas vezes ou até que se encontrasse o chefe da família, visando recuperar os ausentes na primeira visita. As entrevistas seguiram o questionário domiciliar e individual do tipo "perguntas fechadas", no qual se recolheram informações de todos os indivíduos que aceitaram participar do inquérito. $\mathrm{O}$ exame dermatológico para confirmação do critério classificatório constituiu-se na verificação de lesões sugestivas de hanseníase, mediante exame físico sem vestimenta em todos os moradores com mais de sete anos. Os casos considerados suspeitos pela equipe de entrevistadores foram encaminhados ao serviço local de Saúde para exame clínico por especialista.

A caracterização dos domicílios e de seus chefes de família descrita neste trabalho está 
baseada na dicotomização das variáveis independentes em duas categorias: (1) "alto risco" e (0) "baixo risco". Os pontos de corte foram definidos segundo a distribuição de algumas características, de acordo com os resultados do censo de 1980 realizado pelo IBGE, para o município de São Gonçalo, e segundo os parâmetros já estabelecidos anteriormente (Doull et al., 1936; Guinto \& Rodrigues, 1941; Del Favero, 1948; Bechelli, 1949; Rao et al., 1969; Liberatos et al., 1988).
As variáveis idade, grau de escolaridade, sexo, tempo de residência no domicílio investigado e tipo de calçado usado referem-se às informações exclusivas do chefe da família residente há mais de um ano no domicílio. Consideraram-se aglomerados de casas aquelas construídas no mesmo terreno, com cozinha e tanque comuns para mais de uma família. Na variável número de cômodos, não foram computados a cozinha e o banheiro (Quadro 1).

QUADRO 1. Variáveis Estudadas para a Caracterização dos Domicílios e de seus Chefes de Família

\begin{tabular}{|l|l|l|}
\hline \multirow{2}{*}{ Variável } & \multicolumn{2}{c}{ Classes } \\
\cline { 2 - 3 } 1. Tipo de Casa & $\cdot$ aglomerados de casas ou barraco & $\cdot$ casa ou apartamento \\
\hline 2. Área & $\bullet$ menor ou igual a $50 \mathrm{~m}^{2}$ & $\bullet$ mais de $50 \mathrm{~m}^{2}$ \\
\hline 3. Tipo do Piso & $\bullet$ terra ou cimento & $\bullet$ tapete; taco ou cerâmica \\
\hline 4. Água & $\bullet$ nenhum & $\bullet 1$ ou mais pontos de água \\
\hline 5. No de Cômodos & $\bullet 2$ cômodos ou menos & $\bullet 3$ cômodos ou mais \\
\hline 6. No de Pessoas & $\bullet \geq 5$ pessoas & $\bullet 1$ a 4 pessoas \\
\hline 7. Varrer a Casa & $\bullet \leq 1$ vez por semana & $\bullet$ mais de 1 vez por semana \\
\hline 8. Idade & $\bullet$ maior de 40 anos & $\bullet 39$ anos ou menos \\
\hline 9. Escolaridade & $\bullet$ até $1^{\circ}$ grau & $\bullet$ maior que o 2" grau \\
\hline 10. Sexo & $\bullet$ feminino & $\bullet$ masculino \\
\hline 11. Tempo de Residência no Domicílio & $\bullet$ mais de 11 anos & $\bullet 10$ anos ou menos \\
\hline 12. Uso de Calçado & $\bullet$ nenhum ou sandália & $\bullet$ sapato \\
\hline \hline
\end{tabular}

Neste trabalho utilizou-se na estimação das medidas de risco a técnica da regressão múltipla logística (Campos-Filho \& Franco, 1989). O cálculo do intervalo de confiança da razão dos produtos cruzados (R.P.C) foi efetuado para um nível de significância de 0,05 .

A unidade de análise foi definida como o domicílio. A definição de um domicílio com caso de hanseníase foi baseada no estudo de Guinto \& Rodrigues (1941), no qual o domicílio de doente de hanseníase é aquele em que vive um ou mais casos de hanseníase há mais de um ano.
Inicialmente uma análise univariada das 12 variáveis independentes foi processada, comparando-se os domicílios com casos de hanseníase presentes (Grupo I; $\mathrm{n}=137$ ) com os seus respectivos vizinhos (Grupo II; $\mathrm{n}=394$ ) e com os domicílios fora do foco (Grupo III; $\mathrm{n}=395$ ).

As variáveis que apresentaram nível descritivo igual ou menor de que 0.05 nessa etapa foram então reprocessadas por análise multivariada. Os domicílios do Grupo I foram pareados com os seus respectivos vizinhos (Grupo II) e processados pelo método condicional. Para a comparação entre os grupos I e 
III, domicílios que foram sorteados ao acaso, todos localizados em setores onde não havia casos registrados, foi utilizado o método incondicional.

\section{RESULTADOS}

Somente em $12 \%$ dos 532 setores censitários, não foi identificado nenhum caso de hanseníase. O setor censitário assumiu o papel de unidade de estudo dos foco, sendo os domicílios microfocos (Sinneccker, 1976). Constatou-se alto índice focal, com média de 3,15 casos por setor, quando se consideram apenas os casos do registro ativo. Quando se ponderam todos os casos de hanseníase já diagnosticados nesse município, o índice focal é de 4,54 casos/setor censitário. Os setores com casos tratados como focos distribuíram-se como mosaico, concentrando-se na parte mais antiga do município, a sua sede administrativa, área essa fronteiriça do Rio de Janeiro e Niterói (Figura 1).

Nos 137 domicílios selecionados, a freqüência de apenas um caso de hanseníase foi $84 \%$. Dois casos ocorreram em $8 \%$ deles, e também $8 \%$ tinham três ou mais casos. Nesses domicílios, a média de idade do chefe de família foi de 42 anos, e, nos 789 domicílios sem doentes, 40 anos.

Dos 137 domicílios com casos, $94 \%$ dos doentes eram o próprio chefe da família, sendo que $52 \%$ foram classificados como paucibacilares (tuberculóides ou indeterminados), e 48\%, como multibacilares (virchowianos ou dimorfos).

A Tabela 1 mostra o resultado da análise univariada, considerando as diferenças entre as características dos domicílios dos doentes e seus vizinhos, e aqueles fora do foco.

De 531 domicílios dos setores foco, doentes e seus vizinhos, $23,6 \%$, consistiam de casas aglomeradas que dividem a mesma cozinha e lavanderia, contra somente $6,9 \%$ dos domicílios fora foco (R.P.C $=4.12 ; \mathrm{p} \leq 0.00$ ).

Dos domicílios de doentes, $57,6 \%$ são casas de $50 \mathrm{~m}^{2}$ ou menos, enquanto $65,7 \%$ dos vizinhos e $61,8 \%$ dos domicílios fora do foco dispõem de menos de $50 \mathrm{~m}^{2}$. Em $46,7 \%$ dos domicílios de doentes e $46,2 \%$ de seus vizinhos, a terra ou o cimento foi o tipo de piso mais utilizado, contra $57,2 \%$ daqueles fora dos focos (R.P.C $=0.62 ; \mathrm{p}=0.02)$.

Quando se comparou a disponibilidade de pontos de água intradomiciliares, observou-se que $12,5 \%$ dos domicílios de doentes e $14,8 \%$ dos domicílios vizinhos não dispõem de água intradomiciliar, contra $21,5 \%$ (R.P.C $=0.42$; $\mathrm{p}=0.00)$ dos fora dos focos.

Em relação à divisão de suas áreas, os dados mostraram que $30,8 \%$ dos casas de doentes, $46,7 \%$ das vizinhas (R.P.C $=0.58 ; \mathrm{p}=0.03$ ) e $39,4 \%$ dos domicílios fora do foco possuíam no máximo dois cômodos.

Em 45,9\% dos domicílios de doentes de hanseníase residem mais de cinco pessoas, contra $33,3 \%$ nos domicílios vizinhos e $40,8 \%$ naqueles fora do foco.

A limpeza dos domicílios, segundo o relato, o hábito de varrer a casa no mínimo uma vez por semana pelos residentes dos domicílios de doentes foi de apenas 3,6\%, mesma proporção nos domicílios vizinhos, semelhante àquela dos domicílios fora do foco $(4,6 \%)$.

Em resumo, na análise univariada, pareando por local de residência, isto é, Grupo I contra Grupo II (domicílios vizinhos), viver num domicílio com menos de dois cômodos foi o único fator que mostrou associação significativa, com nível de confiança de 0,05 . Na comparação entre o grupo de domicílios de doentes e o Grupo III, ou seja, domicílios fora do foco, destacou-se a associação, com nível de significância de 0,05, de casas do tipo aglomeradas, com piso de terra ou cimento, no lugar de taco ou cerâmica, que apresentam, na grande maioria, mais de um ponto de água no interior da casa.

Quando se compararam as possíveis diferenças das características dos chefes de família de domicílios com doentes de hanseníase com as de seus vizinhos (Tabela 2) e daqueles residentes fora do foco, observou-se freqüência de $72,8 \%$ dos chefes com mais de 40 anos nas família com doentes, de $47 \%$ (R.P.C $=4.32$; $\mathrm{p}<0.00)$ e $52 \%($ R.P.C $=2.57 ; \mathrm{p}=0.00)$ para os chefes vizinhos e residentes fora do foco, respectivamente. 


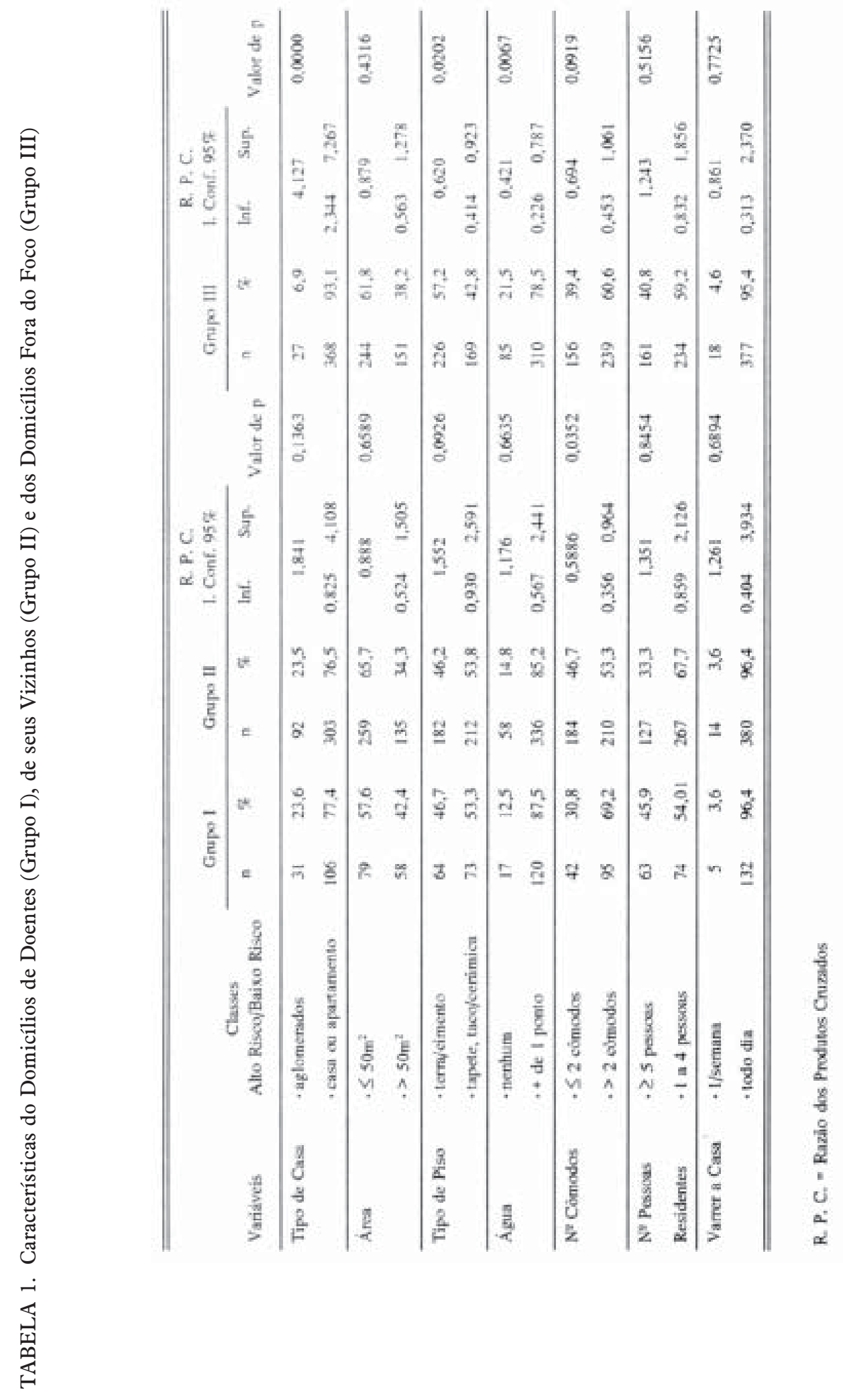


Nos chefes de família dos domicílios com caso de hanseníase, a freqüência de nível de escolaridade acima do Primeiro Grau foi 10,3\%, contra 22,5\% (R.P.C $=2.70 ; \mathrm{p}=0.01)$ nos seus vizinhos e $20,8 \%($ R.P.C $=2.10 ; \mathrm{p}=0.04)$ nos que residem fora do foco.

Nos domicílios com caso, 36,5\% dos chefes de família eram mulheres, enquanto $37,6 \%$ nos domicílios vizinhos e 28,6\% (R.P.C $=1.49$; $\mathrm{p}=0.05$ ) entre os residentes fora do foco eram do sexo feminino.

Quanto à estabilidade atual na residência, $55,6 \%$ dos chefes de família dos domicílios de caso, $42,3 \%$ dos chefes vizinhos (R.P.C $=2.75$; $\mathrm{p}=0.00)$ e $35,7 \%($ R.P.C $=2.46 ; \mathrm{p}=0.00)$ dos residentes fora do foco moram no domicílio há mais de 11 anos.

O hábito de usar sandálias ou, mesmo na maior parte do dia, manter os pés descalços apresentou distribuição semelhante para os três grupos, em torno de $70 \%$.

No processamento multivariado condicional das variáveis (Tabela 3 ) idade do chefe da família, escolaridade, tempo de residência e número de cômodos como diferenciadoras de domicílios e família do Grupo I, domicílios com doentes, em relação aos seus vizinhos (Grupo II), somente se confirmou a associação com a idade (R.P.C $=2.66 ; \mathrm{p}=0.00)$ e o nível de escolaridade $($ R.P.C $=2.54 ; \mathrm{p}=0.03)$.

A Tabela 3 também apresenta os resultados da análise multivariada pelo método incondicional, comparando as características dos chefes de família e dos domicílios com casos de hanseníase em relação àqueles localizados fora do foco. Foram reconfirmados como fatores diferenciais a idade (R.P.C $=2.07$; $\mathrm{p}=0.02$ ), o tipo de casa (R.P.C $=3.95$; $\mathrm{p}=0.00)$ e a disponibilidade de pontos de água intradomiciliar (R.P.C $=0.37 ; \mathrm{p}=0.37)$.

\section{DISCUSSÃO}

A caracterização da extensão dos focos de hanseníase por meio do mapeamento dos domicílios de casos confirmou a tendência de moradia nas áreas centrais e mais antigas do município. Ao mesmo tempo, a ocorrência de casos de hanseníase em mais de $80 \%$ dos setores do município foi fator revelador da existência de um foco de hanseníase já difusamente instalado nessa área urbana, dado compatível com a sua antigüidade do foco (Andrade et al., 1990).

Um dos problemas que deve ser considerado nos estudos de história de caso e controle para identificação de fatores associados na hanseníase é o viés resultante da seleção dos participantes em função da sua probabilidade de inclusão decorrente do tempo de permanência no registro ativo. Neste estudo não podemos afastar esse tipo de obstáculo; entretanto, a inclusão apenas de casos novos diagnosticados não resolveria o problema, porque a detecção de casos se remete àqueles registrados no ano, “incidência de registro" (Fontes, 1967), e não exatamente ao período do diagnóstico nem, muito menos, ao verdadeiro início da doença.

Para o plano amostral considerou-se a prevalência total de registro a mais fiel para a definição dos focos de nosoárea (Sinneccker, 1976) utilizada no estudo das características dos domicílios e dos chefes de família.

A análise multivariada condicional e incondicional apontou de que forma fatores identificados por meio de estudo de associação que não controlam variáveis interferentes podem ser inadequadas para caracterizar os fatores associados. Dentro da área do foco, a diferença do Grupo I com os seus vizinhos, sugerida na análise univariada com o número de cômodos, foi esvaziada na análise condicional pelas variáveis idade e grau de escolaridade.

Para a diferenciação dos domicílios da área do foco daqueles fora do foco, o tempo de residência e o tipo de casa são as variáveis que interferem na explicação da associação com tipo de piso. Se consideramos o tempo de residência como medida do tempo de exposição, essa variável também será interferente para o grau de escolaridade e a freqüência dos chefes de família segundo o sexo, associações essas que não foram confirmadas na análise multivariada incondicional.

$\mathrm{Na}$ área fora do foco observa-se que a idade, o tipo de casa e a disponibilidade de pontos de água intradomiciliar apresentam-se como os prováveis fatores diferenciais em relação às características dos chefes de família e dos domicílios com casos de hanseníase. As casas do tipo aglomerada, utilizando cozinha comum 


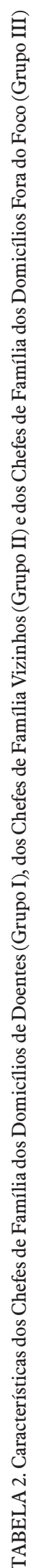

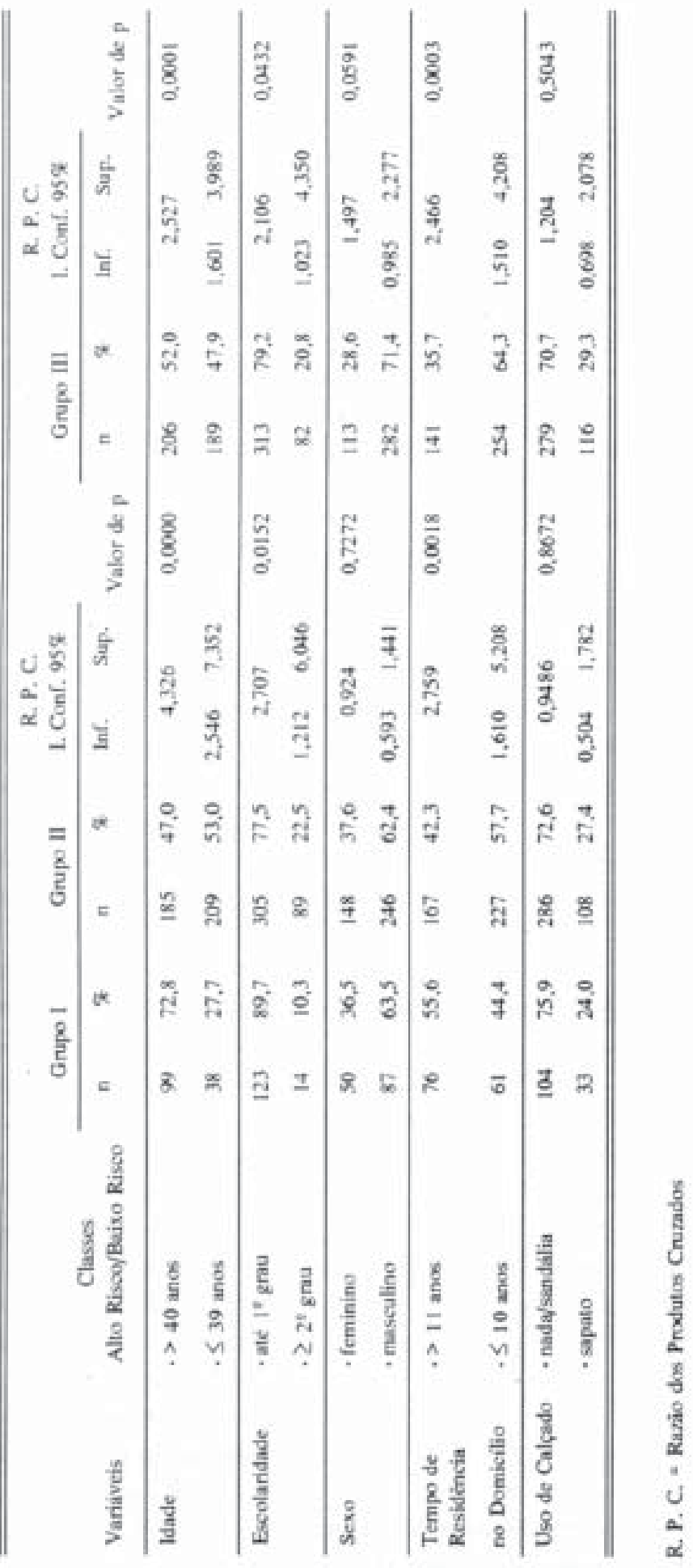


TABELA 3. Comparação das Características dos Domicílios e dos Chefes de Família de Doentes (Grupo I), com os Domicílios e Chefes de Família Vizinhos (Grupo II) e com os Domicílios e Chefes de Família dos Domicílios Fora do Foco (Grupo III)

\begin{tabular}{|c|c|c|c|c|c|c|c|}
\hline \multirow{3}{*}{ Variáveis } & \multirow{3}{*}{$\begin{array}{c}\text { Classes } \\
\text { Alto Risco/Baixo Risco }\end{array}$} & \multicolumn{2}{|c|}{$\begin{array}{c}\text { Grupo II } \\
\text { I. Conf. 95\% }\end{array}$} & \multicolumn{4}{|c|}{$\begin{array}{c}\text { Grupo III } \\
\text { I. Conf. } 95 \%\end{array}$} \\
\hline & & & & \multirow{2}{*}{$\begin{array}{l}\text { Valor de } \\
\qquad \mathrm{p}\end{array}$} & & & \multirow{2}{*}{$\begin{array}{l}\text { Valor de } \\
\mathrm{p}\end{array}$} \\
\hline & & Inf. & Sup. & & Inf. & Sup. & \\
\hline \multirow[t]{2}{*}{ Escolaridade } & - até $1^{\circ}$ grau & \multicolumn{2}{|c|}{2,543} & 0,0360 & \multicolumn{2}{|c|}{1,776} & 0,1662 \\
\hline & $\cdot \geq 2^{\circ} \mathrm{grau}$ & 1,063 & 6,085 & & 0,788 & 4,003 & \\
\hline \multirow[t]{2}{*}{ Idade } & - $>40$ anos & \multicolumn{2}{|c|}{2,661} & 0,0062 & \multicolumn{2}{|c|}{2,071} & 0,0258 \\
\hline & $\cdot \leq 39$ anos & 1,321 & 5,362 & & 1,092 & 3,927 & \\
\hline \multirow{2}{*}{$\begin{array}{l}\text { Tempo de } \\
\text { Residência } \\
\text { no Domicílio }\end{array}$} & - > 11 anos & \multicolumn{2}{|c|}{1,474} & 0,2939 & \multicolumn{2}{|c|}{1,519} & 0,1428 \\
\hline & $\cdot \leq 10$ anos & 0,714 & 3,039 & & 0,868 & 2,659 & \\
\hline \multirow[t]{2}{*}{ Tipo de Casa } & - aglomerados & \multirow{2}{*}{\multicolumn{2}{|c|}{ NS }} & NS & \multicolumn{2}{|c|}{3,950} & 0,0007 \\
\hline & - casa ou apartamento & & & & 1,790 & 8,717 & \\
\hline \multirow[t]{2}{*}{ Tipo de Piso } & - terra/cimento & \multirow{2}{*}{\multicolumn{2}{|c|}{ NS }} & NS & \multicolumn{2}{|c|}{0,872} & 0,6402 \\
\hline & - tapete, taco/cerâmica & & & & 0,491 & 1,548 & \\
\hline \multirow[t]{2}{*}{ Água } & $\cdot$ nenhum & \multirow{2}{*}{\multicolumn{2}{|c|}{ NS }} & NS & \multicolumn{2}{|c|}{0,372} & 0,0310 \\
\hline & $\bullet+$ de 1 ponto & & & & 0,151 & 0,914 & \\
\hline \multirow[t]{2}{*}{ Nº̂modos } & $\cdot \leq 2$ cômodos & \multicolumn{2}{|c|}{0,757} & 0,4368 & \multicolumn{2}{|c|}{0,694} & 0,0919 \\
\hline & - > 2 cômodos & 0,376 & 1,526 & & 0,453 & 1,061 & \\
\hline
\end{tabular}

R. P. C. = Razão dos Produtos Cruzados

NS (Não Selecionada) = Variável não selecionada para a análise multivariada a partir da análise univariada.

e/ou uma única área de lavanderia, mostraram um risco 3,9 vezes maior de ser um domicílio de doente quando comparadas a casas ou apartamentos na área fora do foco. Como as diferenças de morbidade estão associadas a diferentes modos de organização espacial e social, determinando dessa forma diferentes riscos de adoecer para certos grupos sociais, é provável que esses fatores nessa área sejam mais um indicador das condições de vida a que parte da população está submetida do que diretamente do risco de adoecer de hanseníase, reforçando a hipótese de que as características dos domicílios são marcadores da situação social e econômica dos grupos populacionais.
Os contrastes da área fora do foco, como relatado anteriormente, são, dessa forma, indicativos de um assentamento populacional mais recente e não de fatores associados à hanseníase. Verifica-se que a distribuição espacial dos casos de hanseníase acompanhou a organização do município, dado ser um foco estabelecido desde a época de sua origem, caracterizando uma região mais antiga onde se acumulam, ainda que precários, recursos de saneamento.

É somente na análise pareada dos doentes com seus vizinhos, estando então controlados os fatores ambientais da área do foco, que se confirma a associação da idade e do grau de 
escolaridade. Achados esses concordantes com as observações de Doull et al. (1936), e Guinto \& Rodrigues (1941), em que não se registrou qualquer diferença entre as características dos domicílios de doentes de hanseníase e os de não doentes.

A chance de o domicílio ter um doente quando seu chefe de família apresenta baixo nível de escolaridade foi 2,5 vezes maior do que naqueles com maior nível de escolaridade. Considerando a variável grau de escolaridade, utilizada em estudos de outras patologias (Liberatos et al., 1988), como medida do status sócio-econômico, os resultados observados neste estudo, como em outros (Rao et al., 1972; Maine et al., 1988), mostraram que mais de $89 \%$ dos indivíduos portadores de hanseníase têm baixa escolaridade quando comparados com seus vizinhos e residentes fora do foco. Essa constatação conduz à reflexão sobre a atitude dos profissionais de saúde em relação às atividades do controle da hanseníase.

Procedimentos técnicos e recomendações, por vezes complicados, impostos aos pacientes no seu dia-a-dia, tais como receitas com prescrições de medicação auto-administrada em dias alternados, de corticosteróide, ações de prevenção de incapacidades e noções sobre a doença oferecidas sem maior cuidado a um grupo de indivíduos sabidamente com baixo grau de escolaridade, são provavelmente fatores que motivam o afastamento do serviço de saúde, levando-os ao abandono do tratamento.

Os resultados aqui discutidos apontam que o domicílio onde os doentes vivem não é apenas a menor unidade espacial onde se realiza o processo de transmissão do parasito, mas também uma unidade fundamental no estudo da reprodução social (Breilh, 1988), na qual se materializam as relações culturais e econômicas dos grupos em sua coletividade.

O mosaico de setores identificados com a nosoárea mostrou ser uma região antiga, onde já houve mais oportunidades de serem instalados recursos de saneamento. Esses achados são contrários ao observado em Cordova (Doull et al., 1936) onde as áreas mais pobres e mais isoladas eram ocupadas por doentes de hanseníase, desejosos de escapar à detecção e segregação.

Em relação à migração, nesse município ela não parece contribuir de modo relevante mediante a introdução de novas fontes de infecção. Ao contrário, como a endemia já está instalada na área central mais antiga, os imigrantes estão contribuindo para manter o processo de transmissão por meio do aporte de susceptíveis.

Com os determinantes do nível particular próprio do grupo social interagem, no nível individual, a idade e o grau de escola ridade do chefe da família, como fatores que contribuem para explicar a distribuição da hanseníase nessa área do Estado do Rio de Janeiro.

\section{RESUMO}

ANDRADE, V. L. G.; SABROZA, P. C. \& ARAÚJO, A. J. G. Fatores Associados ao Domicílio e à Família na Determinação da Hanseníase, Rio de Janeiro, Brasil. Cad. Saúde Públ., Rio de Janeiro, 10 (suplemento 2): 281-292, 1994.

O objetivo deste estudo foi analisar algumas características dos domicílios e dos chefes de família como possíveis fatores de determinação da hanseníase. Compararam-se unidades domiciliares onde existiam casos de hanseníase com dois grupos de domicílios de não hansenianos. Como grupos de domicílios sem casos de hanseníase, estudaram-se os domicílios vizinhos dos doentes e os domicílios fora dos focos ou áreas sem casos de hanseníase. Todos os domicílios localizamse no município de São Gonçalo, área endêmica urbana na periferia da região metropolitana do Rio de Janeiro. Em um mapa aerofotogramétrico, contendo a descrição dos 584 setores censitários, marcaram-se todos os 2412 casos diagnosticados residentes. Compararam-se os domicílios com casos de hanseníase, Grupo I, com os seus respectivos vizinhos (Grupo II) e com os domicílios fora do foco (Grupo III). Os domicílios do Grupo I foram pareados com os seus respectivos vizinhos (Grupo II) e processados pelo método condicional. Para a comparação entre os grupos I e grupo III, usou-se o método incondicional. O Grupo I comparado com o II apresentou associação entre idade e nível de escolaridade do chefe 
da família. A comparação das características dos chefes de família e dos domicílios com casos de hanseníase em relação aos localizados fora do foco mostrou, como fatores diferenciais, idade, tipo de casa e disponibilidade de pontos de água intradomiciliar. Os autores discutem que os contrastes da área fora do foco são indicativos de um assentamento populacional mais recente e não de fatores associados à hanseníase. Somente na análise pareada dos doentes com seus vizinhos, estando então os fatores ambientais da área de foco controlados, confirmou-se a associação da idade e do grau de escolaridade. Com os determinantes do nível particular próprio do grupo social, interagem, no nível individual, a idade e o grau de escolaridade como fatores que contribuem na morbidade da hanseníase nessa área do Estado do Rio de Janeiro.

Palavras-Chave: Hanseníase; Áreas

Urbanas; Habitação Humana;

Epidemiologia; Estudo de Prevalência

\section{REFERÊNCIAS BIBLIOGRÁFICAS}

ANDRADE, V. L. G., 1990. Características Epidemiológicas da Hanseníase em Área Urbana: Município de São Gonçalo - Rio de Faneiro. Tese de Mestrado, Rio de Janeiro: Escola Nacional de Saúde Publica, Fundação Oswaldo Cruz.

ANDRADE, V. L. G.; SABROZA, P. C.; CASTRO, A. J. W. \& MOTTA, C. P., 1990. Leprosy spread in urban area. Part 1: Epidemiological characteristics of a leprosy endemic urban area: the county of São Gonçalo - Rio de Janeiro Brasil. Hansenologia Internationalis, 15: 24-45.

BANCROFT, H.; GUINTO, R. S.; RODRIGUES, J. N. \& MARQUES, A. P., 1944. A note on familial relationship and the risk of developing leprosy. International Fournal of Leprosy, 12: 79-82.

BECHELLI, L. M., 1949. A importância das causas predisponentes na epidemiologia da lepra. $R e$ vista Brasileira de Leprologia, 17: 175-185.

BREILH, J., 1988. Princípios generales para un nuevo tipo de método en la investigation epidemiológica. In: Epidemiologia Economia, Medicina y Política, pp. 177-191, México: Editorial Premia.

BROWNE, S. G., 1985. The history of leprosy. In: Leprosy (R. C. Hasting, ed.), pp. 53-87, Edinburgh: Churchill Livingstone.
BURGOS, C., 1939. Inquérito epidemiológico. Revista Brasileira de Leprologia, 7: 37-46.

CAMPOS-FILHO, N. \& FRANCO, E. L., 1989. A microcomputer program for multiple logistic regression by unconditional and conditional maximum likelihood methods. American fournal of Epidemiology, 129: 439-444.

CHATURVEDI, R. M., 1988. Epidemiological study of leprosy in Malawani suburb of Bombay. Leprosy Review, 59: 113-120.

DEL FAVERO, W., 1948. O Censo intensivo de Candeias. Arquivo do Servico Nacional de Lepra, 1: 87-235.

DOULL, J. A.; RODRIGUEZ, J. N.; GUINTO, R. \& PLANTILHA, F. C., 1936. A field study of leprosy in Cebu. International Fournal of Leprosy, 4: 141-170.

DOULL, J. A.; GUINTO, R. S.; RODRIGUES, J. N. \& BANCROFT, H., 1942. The incidence of leprosy in Cordova and Talisay, Cebu, Part I. International Fournal of Leprosy, 10: 107-131.

FONTE, J., 1967. Epidemiologia e profilaxia da lepra. Boletim do Serviço Nacional de Lepra, ano XXVI: 31-41.

GEORGE, K.; JONH, K. R.; MULYIL, J. P. \& JOSEPH, A., 1990. The role of intrahousehold contact in the transmission of Leprosy. Leprosy Review, 671: 60-63.

GUINTO, R. S. \& RODRIGUES, J. N., 1941. A field study of Leprosy in Talisay, Cebu. International Fournal of Leprosy, 2: 02-13.

IBGE (Fundação Instituto Brasileiro de Geografia e Estatística), 1980. Censo Demográfico de 1980. Dados Distritais: Rio de Faneiro, 1983. Volume 1, T. 17, Rio de Janeiro: IBGE.

LIBERATOS, P.; LINK, B. G. \& KELSEY, J. L., 1988. The measurement of social class in epidemiology. Epidemiologic Review, 10: 87-121.

MAINE, N.; PONNIGHAUS, J. M. \& FINE, P. E. M., 1988. Problemas in the Analysis of SocioEconomic Risk Factors in Leprosy. XIII International Leprosy Congress Haya, I.L.A. (Mimeo.)

MARSHALL, J. T.; AMAR, D. S. \& RAMESH, H. C., 1981. Prevalence of leprosy among slum dwellers. Leprosy India, 53: 70-83.

MARTINS DE BARROS, J.; QUAGLIATO, R.; VEITIEKA, J.; NETTO, J. P. \& CAMARCO, D. P., 1960. Censo leprótico intensivo de Santa Gertrudes. Revista Brasileira de Leprologia, 28: 157-166.

PONNIGHAUS, J. M.; FINE, P. E. M.; BLISS, L.; SLINEY, I. J.; RADLEY, D. J. \& REES, R. J. W., 1987. The lepra evaluation project (LEP) an epidemiological study of leprosy in northen Malawi. I. Methods. Leprosy Review, 58: 359-375. 
QUAGLIATO, R.; CARLOTTI, C. C.; LIMA, A. T. \& CAMARGO, D. P., 1963. Censo leprológico intensivo Usina Ester. Município de Cosmopólis, Estado de São Paulo. Revista Brasileira de Leprologia, 31: 84-90.

RAO, P. S. S.; KARAT, A. B. A. \& KARAT, S., 1969. Epidemiological studies in leprosy in Gudiyatham Taluk. II: Patterns of familial aggregation of leprosy in endemic area. Leprosy Review, 40: 93-98.

RAO, P. S. S.; KARAT, A. B. A.; KALIAPERUMAL, V. G. \& KARAT, S., 1972. Prevalence of leprosy in Gudiyatham Taluk. South India Part II. Geoghraphical variations. International fournal of Leprosy, 40: 164-170.
SABROZA, P. C., 1991. Organização Social e Endemias. Rio de Janeiro: Escola Nacional de Saúde Publica, Fundação Oswaldo Cruz. (Mimeo.)

SINNECCKER, H., 1976. Epidemic an epizootis process at work. In: General Epidemiology, pp. 153-215, New York: John Willey \& Sons.

TRAUTMAN, J. R., 1984. Epidemiological aspects of hansen's disease. Bulletin of New York Academy Medicine, 60: 722-731. 